\title{
Post epidural puncture delirium resulting from pneumocephalus: An uncommon presenation
}

\author{
Abhishek Wankar, Amit Maheshwari, Sunil Athale
}

\begin{abstract}
A 50-year-old female came with a history of chronic lower back pain radiating to right leg for which she received an epidural steroid injection. Two hours later, she was found to in a delirious state with bilateral ptosis. Magnetic resonance imaging of the brain was suggestive of ventricular pneumocephalus in frontal and temporal horns of right lateral ventricles. She was managed conservatively with oxygen inhalation. Patient's sensorium improved after $72 \mathrm{~h}$.
\end{abstract}

Key words: Epidural, magnetic resonance imaging, ventricular pneumocephalus

\section{INTRODUCTION}

Epidural steroid injections are frequently employed in the management of degenerative conditions of the lumbar spine including radiculopathy. ${ }^{[1]}$ Despite a known low morbidity, significant complications can occur. Recently, we came across a case where the patient developed delirium and ptosis following an epidural steroid injection

\section{CASE REPORT}

The 50-year-old female came with a history of lower backache radiating to the lateral aspect of the right thigh for last 2 years. For last 2 months, she was unable to walk more than 10 steps due to aggravating back pain with each step. Magnetic resonance imaging (MRI) of the lumbosacral spine showed degenerative changes with annular tear from second to fifth intervertebral space.

\begin{tabular}{|l|l|}
\hline \multicolumn{2}{|c|}{ Access this article online } \\
\hline Quick Response Code: & Website: \\
\hline & www.jnaccjournal.org \\
\cline { 2 - 2 } & \\
\hline
\end{tabular}

A lumbar epidural was performed with the patient in right lateral decubitus with thighs flexed, and neck flexed forward. The L4-L5 interspace was identified clinically. A 20-gauge quincke needle was inserted via a midline approach. The epidural space was reached via the loss-of-resistance technique. Aspiration was negative for cerebrospinal fluid or haematologic products. Epidural triamcinolone acetonide and bupivacaine $4 \mathrm{ml}$ were injected along with $6 \mathrm{ml}$ distilled water in L4-L5 disc space. Two hours later, patient became delirious with bilateral ptosis. Patient was uncooperative for a thorough evaluation of pupils, fundus and eye movements. In view of the temporal relation of the symptoms to the epidural injection, an iatrogenic cause was suspected. However, a preexisting unrecognised intracranial lesion was also under considerations. The MRI-brain imaging including gradient recalled echo (GRE) sequencing was requested. Her laboratory parameters including serum electrolytes, complete haemogram, urine routine microscopy, electrocardiogram and chest X-ray were within normal limits. Patients' haemodynamic and oxygen saturation remained stable throughout her hospital stay.

Magnetic resonance imaging-brain done $3 \mathrm{~h}$ after the procedure showed mild ballooning of frontal and temporal horns of the right lateral ventricles on T1-weighted images [Figure 1]. T2-weighted images showed well-circumscribed hypointensities in frontal

Department of Neurology, SAIMS, Indore, Madhya Pradesh, India

Address for correspondence: Dr. Abhishek Wankar, Department of Neurology, SAIMS, Sawer Road, Indore - 452 010, Madhya Pradesh, India.

E-mail: abhishek.wankar@gmail.com 
horns of both lateral ventricles more prominent on the right [Figure 2]. Diffusion weighted images and fluid attenuation inversion recovery sequences were normal ruling out any acute inflammatory or ischaemic lesion. Magnetisation transfer ratio images showed similar hypointensities as on T2-weighted [Figure 3]. Abnormalities on GRE images were striking and unexpected showing multiple hypointensities (so called "blooming") in bilateral frontal horns on lateral ventricle more prominent on the right, temporal horn of right ventricle with mild effacement of anterior perimesencephalic cistern on the right side. After neuroradiological discussion, MRI-brain was interpreted as ventricular as well as parenchymal pneumocephalus.

Patient was treated conservatively with oxygen inhalation at the rate of $10 \mathrm{~L} /$ min with a polymask. Gradually the patient recovered from her delirium and the ptosis has also disappeared over the next $72 \mathrm{~h}$. A repeat MRI-brain done 6 days after the ictus showed complete radiological resolution [Figure 4].

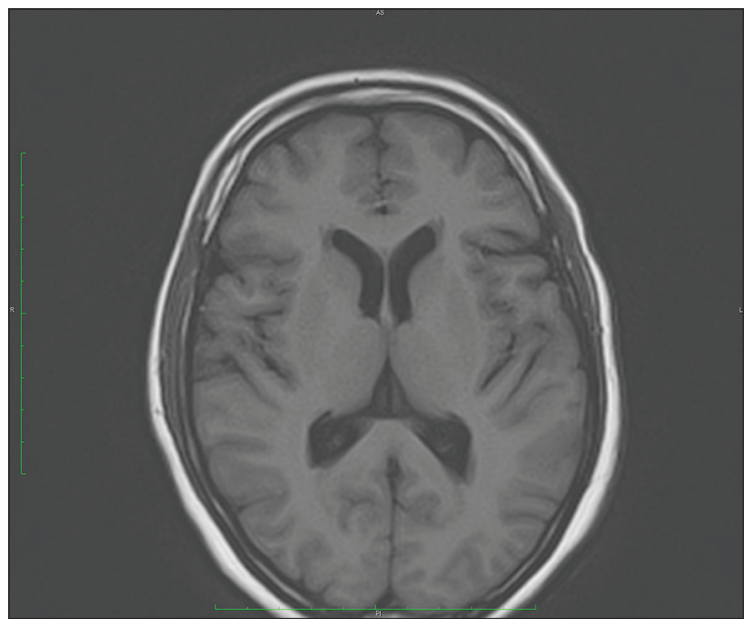

Figure 1: T1-weighted image showed no abnormality

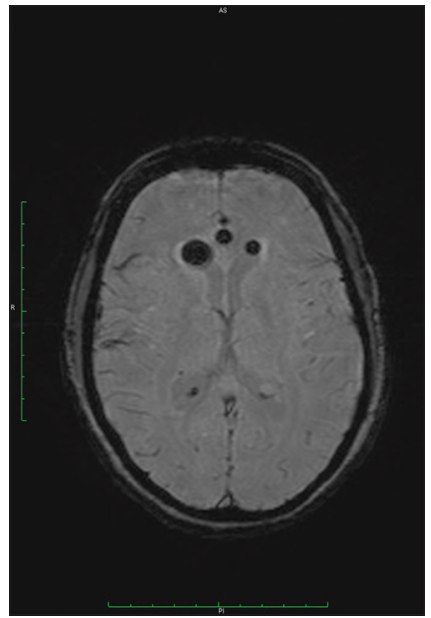

Figure 3: Susceptibility weighted imaging showing blooming

\section{DISCUSSION}

Epidural injections are not free of complications. In literature, aseptic meningitis, arachnoiditis, and bacterial meningitis and conus medullaris syndrome have been reported. ${ }^{[2]}$ Pneumocephalus is an uncommon complication after epidural injection. Various authors have previously described pneumocephalus following an epidural puncture. ${ }^{[3-10]}$ However, unlike our case, headache has been reported as a prominent symptom in all the cases. Also, delirium as a presenting symptom of pneumocephalus is uncommon. The only similar case report from India was by Sweni et al..$^{[6]}$ However, the patient described by Sweni et al., developed pneumocephalus following a cervical puncture and air travel that was not the case in our patient. Brar et al. have highlighted the value of GRE sequence in the diagnosis of air in brain parenchyma or ventricles. This is corroborated in our case as intraparenchymal air was missed in other sequences. Besides, we would like to stress that the so

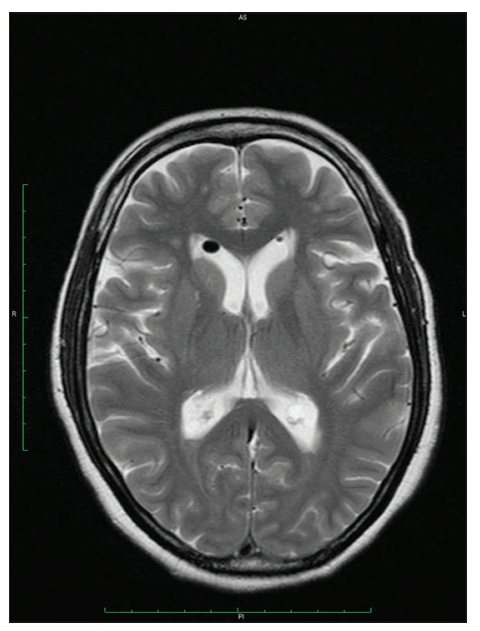

Figure 2: T2-weighted image showing intraventricular hypointensity in frontal horn of right ventricle

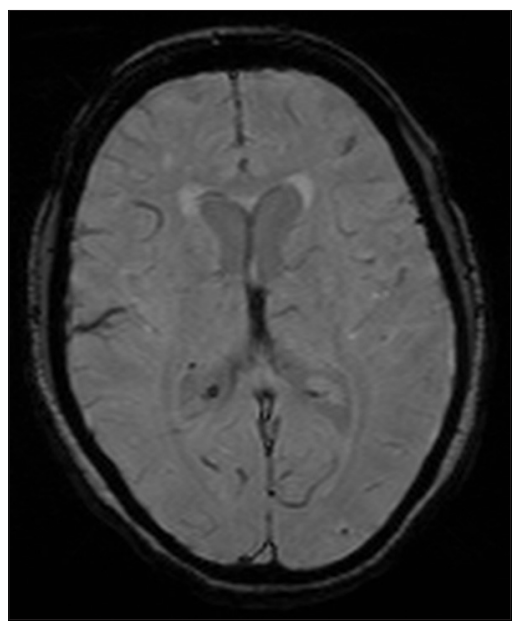

Figure 4: Susceptibility weighted imaging after 6 days showing complete resolution 
called blooming in GRE does not necessarily mean blood or calcium as we learned from this case. ${ }^{[11]}$

Pneumocephalus may result from accidental dural puncture and develops from the injection of air into subdural or subarachnoid space followed by cranial migration. ${ }^{[12]}$ Air introduced into the subdural space is painful and reaches the ventricles rapidly, because of its low pressure and diminished capacitance. The appearance of other symptoms, such as signs focal neurologic deficits including cranial nerve palsies may develop depending on the spread and extent of intracranial air. ${ }^{[13]}$ This may explain development of ptosis in our case.

The presence of pneumocephalus should be considered in any unexplained neurological presentation especially if GRE sequence in MRI shows blooming.

It is well-known that air in the magnetic field has very low magnetic susceptibility and can cause signal loss on MRI, especially on GRE sequence. As in our case patient, this loss of signal can be used to diagnose the presence of subtle intracranial air that is prominently seen on T2-weighted GRE images. The symptoms of pneumocephalus following an epidural injection can be diverse and hence a high degree of suspicion is essential for further work up.

Pneumocephalus has a tendency to resolve spontaneously, and usually it is absorbed after a few days. However, high inspired oxygen concentration can hasten its absorption. Increasing the inspired oxygen reduces the partial pressure of nitrogen in the blood, and by creating a greater concentration gradient for nitrogen, it fastens the intracranial air collection to diffuse into the blood stream. ${ }^{[14]}$

\section{CONCLUSION}

This is a MRI documented case of postepidural puncture intraventricular and intraparenchymal pneumocephalus presenting with delirium as a prominent symptom. Our case highlights the importance of high index of suspicion for such complications.

\section{REFERENCES}

1. Sung MS. Epidural steroid injection for lumbosacral radiculopathy. Korean J Radiol 2006;7:77-9.

2. Abram SE, O'Connor TC. Complications associated with epidural steroid injections. Reg Anesth 1996;21:149-62.

3. Syed SF, Garcon E. A case of diffuse subarachnoid pneumocephalus after epidural injection. OMICS J Radiol 2013;2:120.

4. Verdun AV, Cohen SP, Williams BS, Hurley RW. Pneumocephalus after lumbar epidural steroid injection: A case report and review of the literature. A A Case Rep 2014;3:9-13.

5. Nistal-Nuño B, Gómez-Ríos MÁ. Case Report: Pneumocephalus after labor epidural anesthesia. F1000Res 2014;3:166.

6. Sweni S, Senthilkumaran S, Balamurugan N, Thirumalaikolundusubramanian P. Tension pneumocephalus: A case report with review of literature. Emerg Radiol 2013;20:573-8.

7. Nafiu OO, Urquhart JC. Pneumocephalus with headache complicating labour epidural analgesia: Should we still be using air? Int J Obstet Anesth 2006;15:237-9.

8. Hawley JS, Ney JP, Swanberg MM. Subarachnoid pneumocephalus from epidural steroid injection. Headache 2005; $45: 247-8$.

9. Sherer DM, Onyeije CI, Yun E. Pneumocephalus following inadvertent intrathecal puncture during epidural anesthesia: A case report and review of the literature. J Matern Fetal Med 1999;8:138-40.

10. Ash KM, Cannon JE, Biehl DR. Pneumocephalus following attempted epidural anaesthesia. Can J Anaesth 1991;38:772-4.

11. Brar R, Prasad A, Sharma T, Khosla VK. Pneumocephalus on MRI: Use of gradient imaging. Indian J Neurotrauma 2011;8:59-60.

12. Barbosa FT, Cunha RM, Rocha AP, Silva Júnior HJ. Intraventricular pneumocephalus after accidental perforation of the dura mater: Case report. Rev Bras Anestesiol 2006;56:511-7.

13. Gómez-Ríos MÁ, Fernández-Goti MC. Pneumocephalus after inadvertent dural puncture during epidural anesthesia. Anesthesiology 2013;118:444.

14. Dexter F, Reasoner DK. Theoretical assessment of normobaric oxygen therapy to treat pneumocephalus: Recommendations for dose and duration of treatment. Anesthesiology 1996;84:442-7.

How to cite this article: Wankar A, Maheshwari A, Athale S. Post epidural puncture delirium resulting from pneumocephalus: An uncommon presenation. J Neuroanaesthesiol Crit Care 2016;3:43-5.

Source of Support: Nil, Conflict of Interest: None declared. 